\title{
NA DIREÇÃO DE UMA MODELAGEM DA INFORMAÇÃO DA CIDADE $(\mathrm{CIM})^{1}$
}

\author{
TOWARDS CITY INFORMATION MODELING
Fabiano Rogerio Corrêa fabiano.correa@usp.br \\ Departamento de Engenharia de Construção Civil (USP) \\ Eduardo Toledo Santos \\ Departamento de Engenharia de Construção Civil (USP) \\ etoledo@usp.br
}

\begin{abstract}
Resumo
Aproveitando os esforços, tanto da indústria dentro do paradigma da Modelagem da Informação da Construção (BIM) em estender o esquema de dados Industry Foundation Classes (IFC) para abarcar projetos de infraestrutura, quanto da academia com trabalhos de integração entre o BIM e os Sistemas de Informação Geográficos (SIG), poder-se-ia considerar a relevância deste cenário no desenvolvimento de uma futura Modelagem da Informação da Cidade (City Information Modeling - CIM). Este cenário traduz-se na busca pelo modelo mais apropriado na captura da semântica e da representação geométrica dos objetos pertinentes ao escopo de uma cidade. A semântica é a parte mais relevante, já que permite a aplicação do modelo a diferentes tipos de análises e simulações. Este artigo irá tratar especificamente das iniciativas dentro da buildingSMART para introduzir estradas, túneis, pontes e terraplanagem ao IFC4, estando em comum acordo com as especificações CityGML e InfraGML/LandXML da Open Geospatial Consortium (OGC). Deste modo, será discutida a literatura direcionada à integração entre BIM e SIG para analisar o impacto destas soluções para uma futura CIM. O trabalho consiste de uma discussão teórica, de caráter exploratório, para entender os possíveis caminhos para o desenvolvimento do CIM.
\end{abstract}

Palavras-chave: BIM. CIM. IFC.

\begin{abstract}
Following efforts from industry on the Building Information Modeling (BIM) paradigm in extending Industry Foundation Classes (IFC) to infrastructure, and from academia with studies on integration of BIM and Geographical Information Systems (GIS), it is considered the relevance of this scenario in the future development of a City Information Modeling (CIM). This scenario translates in the search for the appropriate model that captures the semantics and geometric representation of objects that belongs to the context of a city. Semantics is the most important part, as it allows for the application of the model to different types of analysis and simulations. This article will treat specifically of the initiatives of the buildingSMART to introduce roads, tunnels, bridges, and earthwork in IFC4, which are in common accord with CityGML and InfraGML/LandXML from Open Geospatial Consortium (OGC). In this manner, it will be discussed the literature focused in the integration of BIM and GIS to analyze the impact of these solutions to the future of CIM. The work constitutes itself in a theoretical discussion, of exploratory character, to understand the possible ways in the development of CIM.
\end{abstract}

Keywords: BIM. CIM. IFC.

\footnotetext{
${ }^{1}$ CORREA, F. R.; SANTOS, E. T. Na direção de uma modelagem da informação da cidade (CIM). In: ENCONTRO BRASILEIRO DE TECNOLOGIA DE INFORMAÇÃO E COMUNICAÇÃO NA CONSTRUÇÃO, 7 , 2015, Recife. Anais... Porto Alegre: ANTAC, 2015.
} 


\section{7}

\section{TIC2015}

\section{INTRODUÇÃO}

Aproveitando os esforços, tanto da indústria dentro do paradigma da Modelagem da Informação da Construção (BIM) (EASTMAN et al., 2011) em estender o Industry Foundation Classes (IFC) (ISO, 2013) para abarcar projetos de infraestrutura, quanto da academia com trabalhos de integração entre o BIM em nível de edificação e os Sistemas de Informação Geográficos (SIG) em nível de bairros e cidades, poder-se-ia considerar a relevância deste cenário no desenvolvimento de uma futura Modelagem da Informação da Cidade (City Information Modeling - CIM).

O BIM, que tem como uma de suas tecnologias básicas a modelagem 3D paramétrica para o projeto e a construção, tem se consolidado na área da Arquitetura, Engenharia e Construção (AEC), permitindo um fluxo de trabalho colaborativo e mais eficiente tanto em empreendimentos de edificações quanto de infraestrutura pesada. $O$ esquema de dados IFC, promovido pela buildingSMART, tornou-se um padrão internacional de fato para consolidar a interoperabilidade durante todo ciclo de vida da edificação.

Já um SIG é "um sistema computacional capaz de arranjar, armazenar, manipular e exibir informação referenciada geograficamente" (KARIMI; AKINCI, 2010). Integra operações em bancos de dados e análise estatística, com visualizações únicas em um mapa. O primeiro sistema deste tipo foi desenvolvido e lançado na década de 1960, no Canadá (FISHER; MacDONALD, 1979). Desde o surgimento dos primeiros SIGs, a tecnologia da informação e comunicação (TIC) tem sido empregada com sucesso para integrar dados reais de naturezas distintas, georreferenciados, permitindo análises quantitativas e qualitativas de vastas extensões territoriais. Na maior parte das implementações, os SIGs ainda funcionam sobre dados bidimensionais, embora existam iniciativas empregando modelos tridimensionais.

Mesmo tendo sido concebidos com propósitos bastante distintos, ambos os modelos apresentam objetos comuns ao lidar com a infraestrutura. Desde cedo foi reconhecida a importância de haver uma integração maior entre os softwares e modelos que lidam com o BIM e o SIG (KARIMI; AKINCI, 2010). Portanto este cenário mostra-se relevante para o desenvolvimento de uma futura Modelagem da Informação da Cidade (CIM) que, pela própria designação, transcende a mera representação geométrica tridimensional de edifícios, ruas, e demais elementos urbanos.

O CIM considerado aqui está longe de ser apenas um modelo virtual e tridimensional detalhado do espaço urbano, apesar de existirem iniciativas neste sentido. Antes, consonante ao BIM, pretende-se que o CIM seja um novo paradigma para se pensar e gerir o desenvolvimento urbano, que adota um amplo espectro de ferramentas computacionais para concretizar fluxos de trabalho cooperativos e integração de informação heterogênea; que use não apenas as atuais ferramentas do TIC, mas que eventualmente empregue análises do tipo Big Data (BERMAN, 2013).

O termo CIM já foi empregado na literatura científica, mas está longe de estar estabelecido. Pode-se até confundir a ideia de um CIM com os trabalhos propostos no contexto das Smart Cities ou Cidades Inteligentes. Entende-se que um CIM é tanto um elemento quanto uma filosofia que será incorporada no desenvolvimento das Smart Cities do futuro.

Designando Modelagem da Informação da Cidade, o termo apareceu pouco na literatura e, mesmo assim, o conteúdo de tais modelos está longe de ser acordado. Alguns adotam uma perspectiva que engloba muitas visões de uma cidade: arquitetônica, social, econômica, etc. (STOJANOVSKI, 2013). Outros focam nas ferramentas necessárias para lidar com a escala ou tamanho dos modelos (GIL; ALMEIDA; DUARTE, 2011). E também existem proposições na mesma filosofia do BIM para alcançar o CIM (XU et al., 2014).

Este trabalho irá se restringir ao foco da modelagem da informação pertinente ao CIM, já 
que o assunto é multidisciplinar e extremamente amplo. A modelagem da informação determina a semântica existente nos modelos e acaba por promover ou inibir análises e simulações que são possíveis a partir dos modelos.

Mais especificamente, este artigo irá tratar das iniciativas dentro da buildingSMART para introduzir modelagem de infraestruturas (pontes, rodovias, túneis) no IFC, fará uma comparação entre a modelagem de dados no IFC e no CityGML (modelagem de dados para o SIG, determinado pela Open Geospatial Consortium ou OGC), no contexto da literatura direcionada à integração entre BIM e SIG, e analisará o impacto destas soluções para uma futura Modelagem da Informação da Cidade, apontando para uma ideia particular de CIM.

O trabalho consiste de uma discussão teórica, de caráter exploratório, para entender os possíveis caminhos para o desenvolvimento do tipo de CIM considerado.

\section{A BUILDINGSMART ${ }^{2}$ E O TRATAMENTO DE INFRAESTRUTURA NO IFC4}

A buildingSMART, estabelecida em 1996 sob o nome de International Alliance for Interoperability, nasceu de uma aliança entre empresas privadas, organizada pela Autodesk para provar os benefícios da interoperabilidade entre as diferentes soluções de software existentes na indústria da construção. Seu nome atual foi adotado a partir de 2008, e desde então tem expandido sua atuação também para a indústria da infraestrutura. Esta organização promove a ideia do open BIM com base em diversos padrões, o mais importante deles sendo o IFC.

\subsection{IFC4}

O IFC é um modelo de dados que procura representar todos os componentes ou objetos relevantes ao ambiente construído. Através do IFC, os empreendimentos são representados digitalmente e os modelos podem ser trocados entre diferentes plataformas e ferramentas de software, promovendo os fluxos de trabalho e trocas de informações na indústria da construção. Atualmente consiste em 766 entidades, que vão desde a caracterização do local a ser empreendido até os elementos que representam paredes e janelas de uma edificação.

Conforme indicado na Figura 1, as classes do IFC são divididas em quatro camadas: recursos, núcleo, interoperabilidade e domínio. Todas as instâncias das classes dentro das três camadas mais acima são derivadas da classe mais abstrata IfcRoot e possuem um atributo de identificação global única (GloballD), dentre outros. Diferentemente, a camada de recursos está sempre associada a outras classes, e provê, por exemplo, geometria aos produtos da construção.

O IFC4 foi publicado em março de 2013, atualizando o padrão ISO 16739. A esta altura, o IFC já é um padrão de fato na indústria e todos os grandes fabricantes de software possuem uma interface com esse formato.

A última versão do IFC promoveu alterações em seus principais elementos, ligados à arquitetura, sistemas prediais e estrutura. Esses elementos estão com novas características geométricas e paramétricas, possibilitando novos fluxos de trabalho dentro do BIM, incluindo trocas de modelos 4D e 5D. Também possibilitam a interoperabilidade entre BIM e SIG, promovem melhorias em simulações térmicas, e permitem a extensão do padrão IFC para infraestrutura e outras partes do ambiente construído (LIEBICH, 2013).

Juntamente com o IFC, está o MVD (Model View Definition) que resumidamente consiste num subconjunto mínimo que estabelece os elementos de uma troca de informações específica entre aplicativos de profissionais da construção. O modelo IFC é vasto e 
complexo, e as trocas são focadas em elementos particulares. O MVD permite simplificar este tipo de troca. Um arquivo mvdXML, juntamente com o esquema IFC, especifica toda a informação necessária do MVD. Atualmente estão publicados o IFC4 Reference View e o IFC4 Design Transfer View, que substituem o IFC2x3 Coordination View V2.0, relacionado às trocas de modelo durante a coordenação de projeto (buildingSMART, 2015a).

Figura 1 - Arquitetura do Modelo de Dados do IFC4

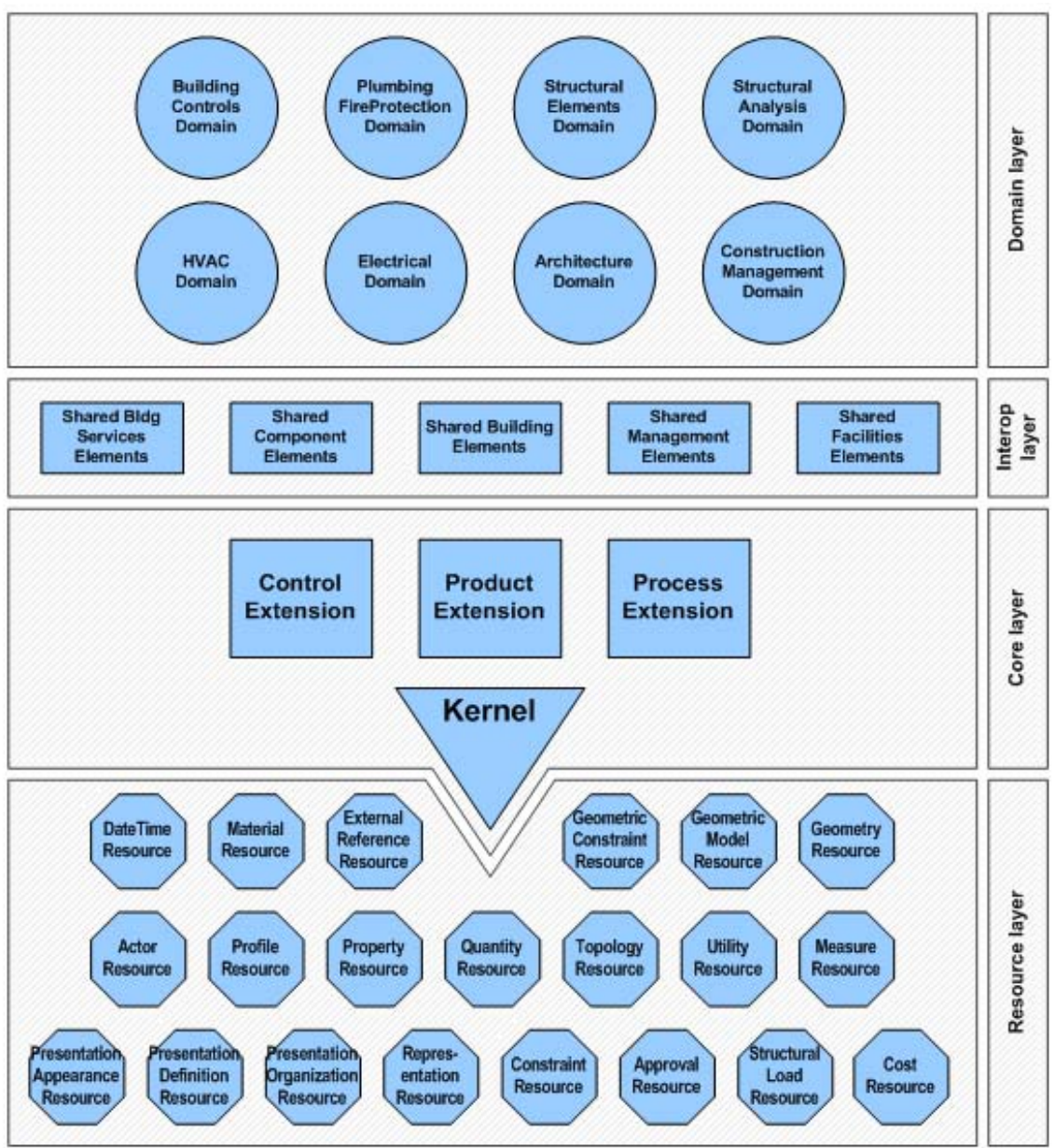

Fonte: buildingSMART, 2015a.

\subsection{Expansão do IFC4 para Infraestruturas}

Os projetos de expansão do modelo de dados IFC4 para incorporar as informações pertinentes ao ciclo de vida de obras de infraestrutura específicas, tais como pontes, estradas e túneis, vem sendo desenvolvidos a cerca de uma década. O desenvolvimento tem sido local por determinados Chapters da buildingSMART, em colaboração com universidades e indústrias.

No entanto, não existe uma documentação centralizada do progresso destes projetos, apenas artigos científicos e apresentações no contexto dos Chapters locais acerca dos mesmos. A proposta de criação de um esquema mais geral, que englobaria parte da informação comum a todas as infraestruturas, o IfcAlignment partiu da sugestão do Prof. Andre Bormann da TUM na Alemanha, e alguns especialistas da Bentley em setembro de 2013 (MOON, 2015). Aparentemente, com o lançamento oficial do IfcAlignment para lidar com infraestrutura em geral, projetos como o IfcBridge, IfcRoad e o IfcTunnel serão revistos 
para estarem alinhados com o novo esquema de dados expandido. A Figura 4 dá um panorama das intenções e dos projetos em andamento dentro da buildingSMART.

Na semântica do IFC4, os componentes da construção são categorizados em elementos físicos (IfcElement) e elementos espaciais (IfcSpatialElement). Todo projeto de extensão do IFC4 para infraestrutura envolverá a criação de componentes específicos derivados destas duas classes. Além disso, as classes IfcCivilElement e IfcCivilElementType foram criadas como pontos de entrada na hierarquia da modelagem dos dados para as futuras classes vinculadas à infraestrutura. A seguir é apresentada uma síntese dos projetos de infraestrutura que já foram desenvolvidos.

\subsubsection{IfcBridge}

A proposta para a criação de um modelo de informação para pontes foi feita em 2002. Em 2004, apareceu a primeira versão do modelo (V1). O modelo V2 em 2006 estava totalmente integrado ao IFC $2 \times 3$, que ainda hoje é o esquema IFC implementado na maior parte dos aplicativos BIM no mercado. Em 2013 saiu a V3, integrada com o IFC4.

O trabalho de desenvolvimento foi encabeçado por Eric Lebegue, do Chapter francês da buildingSMART (ARTHAUD; LEBEGUE, 2002). Em paralelo, um grupo japonês também desenvolveu um modelo de ponte de concreto armado baseado em IFC (YABUKI et al., 2006).

As duas iniciativas deram origem ao atual trabalho com o IfcBridge. A Figura 2 ilustra os elementos presentes na estrutura geral da ponte e em um corte transversal.

Figura 2 - A estrutura geral da ponte e corte transversal.

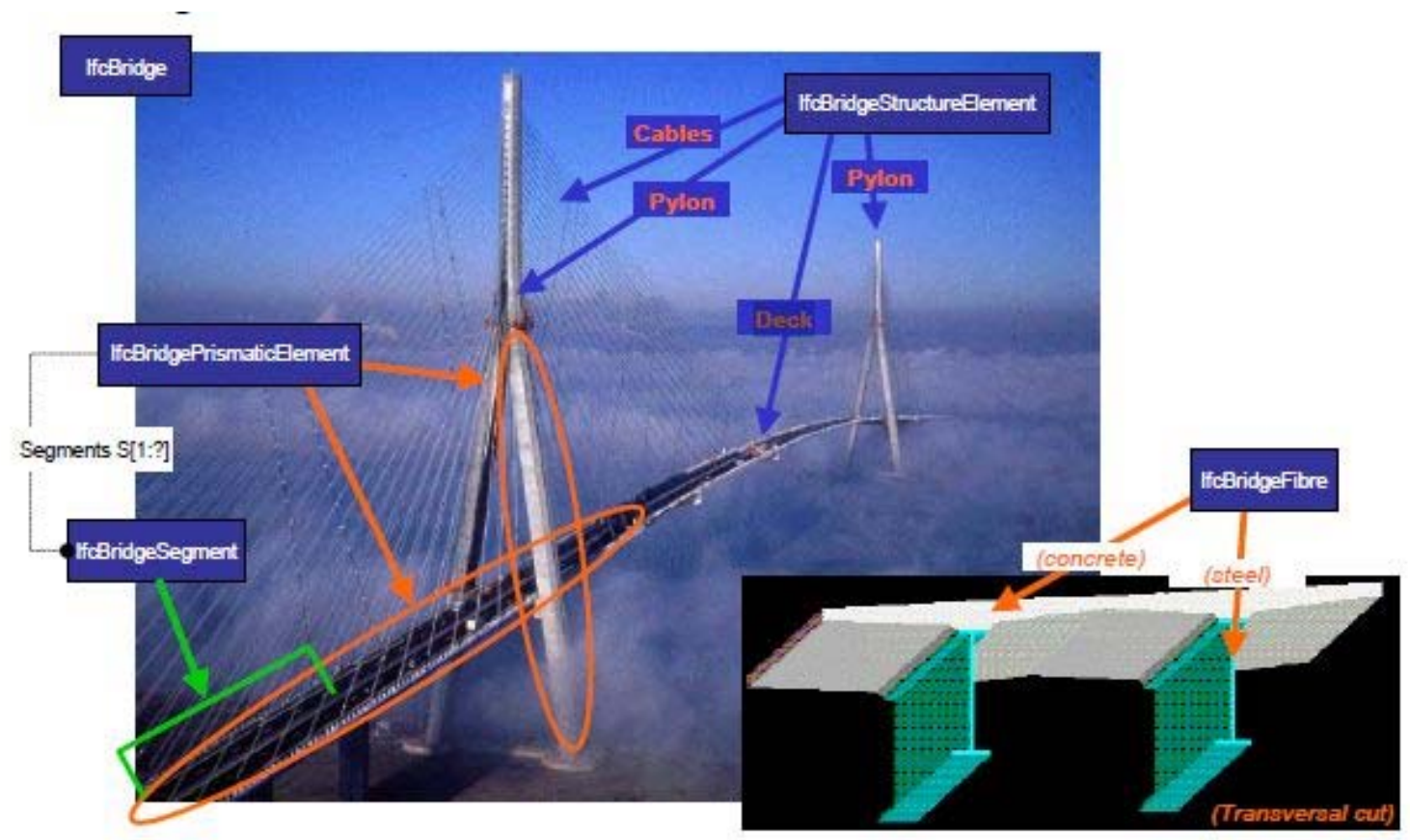

Fonte: YABUKI et al., 2006.

\subsubsection{IfcRoad}

O modelo de informação para estradas vem sendo desenvolvido em duas frentes: o modelo coreano e o proposto pela V-Con. Ambos os projetos almejam obter modelos que não 
apenas representam estradas, mas também pontes (V-Con) ou pontes e túneis (coreano).

No projeto desenvolvido no Instituto Coreano de Tecnologia da Construção (KICT), iniciado em 2012, o escopo restringe-se à informação pertinente da fase de projeto detalhado da estrada, drenagem e facilidades subsidiárias (LEE; KIM, 2011). Não são tratadas as fases de planejamento, construção e manutenção das rodovias nem as disciplinas de arquitetura, mecânica, elétrica, comunicação e paisagismo das mesmas. Desse modo, a informação consistiria basicamente na representação da forma geométrica dos objetos e de seus componentes e atributos básicos. Em 18 de março de 2014, o desenvolvimento deste projeto já estava alinhado com a proposta do IfcAlignment.

$\mathrm{Na}$ V-Con, partiu-se do CityGML, e buscou-se um nível de detalhamento mais adequado para representar os elementos da construção. O modelo IFC representaria um LoD 5 no CityGML.

\subsubsection{IfcTunnel}

Também existem dois projetos independentes para a criação de modelos de túneis, um liderado pela Ruhr University Bochum, na Alemanha (STASCHEIT et al., 2013), e outro pelo IAI-Japão em conjunto com a Universidade de Osaka (YABUKI et al., 2007).

No primeiro, a extensão do IFC para modelar túneis consiste de três sub-domínios: o solo, o túnel e o equipamento de escavação e retenção do solo.

\subsubsection{IfcAlignment}

Deduz-se que, com o desenvolvimento destes projetos pilotos, existiam elementos comuns nestas modelagens: a informação do alinhamento das diversas seções de uma ponte, estrada ou túnel.

Figura 3 - Arquitetura do modelo de dados IFC adaptado para Infraestrutura

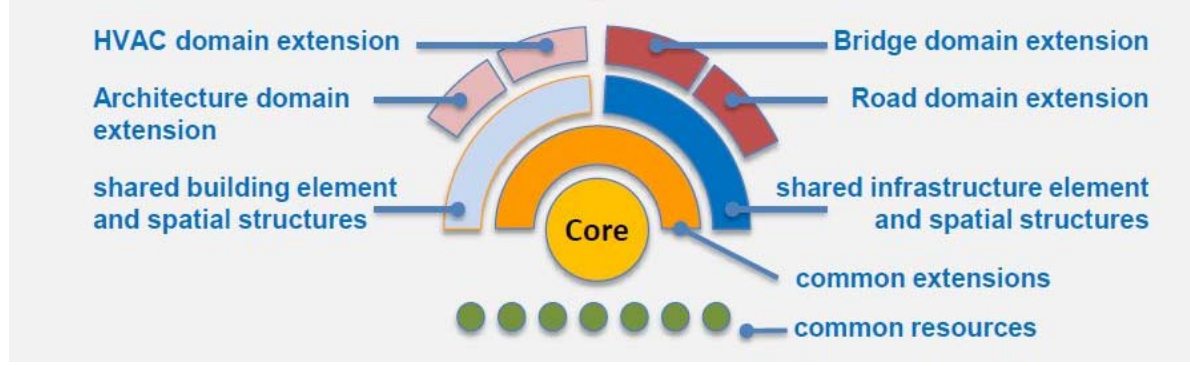

Fonte: LIEBICH, 2012.

Comparando a Figura $1 \mathrm{com}$ a Figura 3, tem-se que, em linhas gerais, a alteração na arquitetura original do IFC4 está nas duas últimas camadas, com o acréscimo de elementos de infraestrutura e estruturas espaciais que são compartilhados pelas extensões de domínio associadas a pontes e estradas - da mesma maneira que existem elementos da edificação e estruturas espaciais compartilhadas para as extensões de domínio ligadas, por exemplo, à arquitetura e à AVAC (Aquecimento, Ventilação e Ar-Condicionado). Como será apresentado mais adiante, acréscimos em outras camadas muitas vezes são necessárias, como no caso do IfcAlignment.

Recentemente, entre 26 de janeiro e 6 de março de 2015, foi colocado para consulta o IfcAlignment, vital para expandir o IFC4 para infraestrutura. A ideia é adicionar subdomínios 
vinculados à infraestrutura, como IfcRoad, IfcTunnel, IfcBridge e IfcAlignment aos outros domínios presentes no IFC, como AVAC, Arquitetura, e assim por diante.

Internamente referido como P6, o projeto "IFC Alignment" é a primeira extensão do IFC para Infraestrutura feita pela buildingSMART.

O alinhamento é "um sistema de referência associado a construção lineares, tais como estradas, trilhos e pontes que é usado para posicionar elementos ao longo de um alinhamento". Ele é obtido por meio da combinação de técnicas tradicionais em 2D com novas abordagens em 3D. Tem-se um alinhamento horizontal e um vertical, juntamente com seções do perfil desta infraestrutura.

Figura 4 - Módulos de infraestrutura para extensão do IFC.
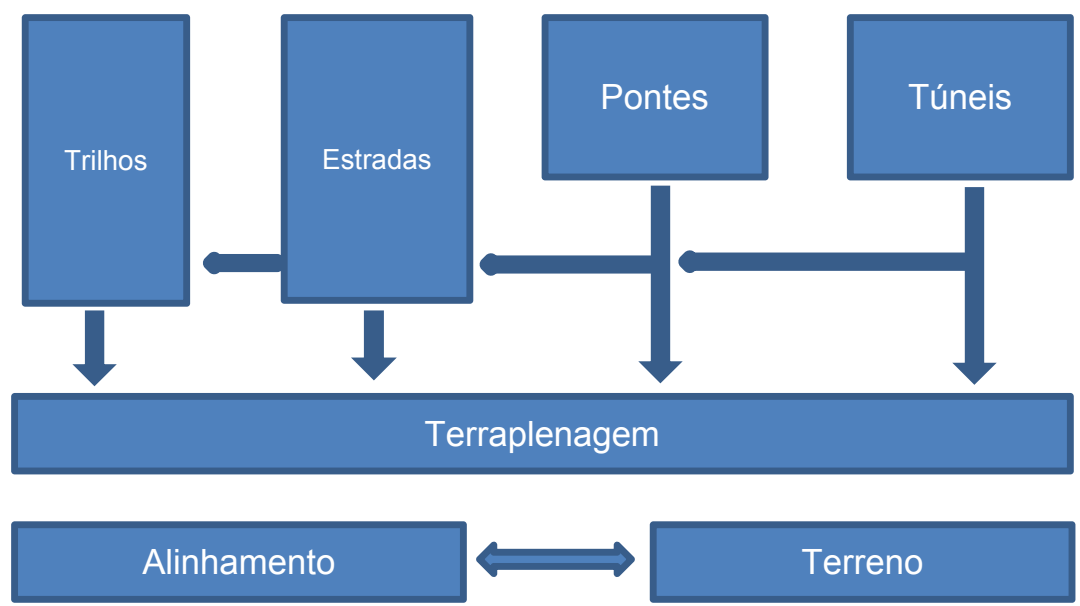

Sistema de coordenadas referenciais, referenciamento linear \& contexto espacial

Fonte: adaptado de buildingSMART, 2015b

A arquitetura do IFC4 foi alterada em três esquemas: Product Extension (Core Layer) e Geometric Constraint Resource e Geometry Resource (Resource Layer). O IfcAlignment entra dentro do IfcProductExtension, junto ao IfcGrid, ambos como instâncias da classe abstrata IfCPositioningElement. Ligados ao IfcAlignment tem-se dois recursos de restrição geométrica, relativos aos alinhamentos horizontal e vertical 2D.

No sentido de prover a geometria adequada para representação da infraestrutura, os tipos de segmentos para o alinhamento horizontal previstos são linha, arco circular e clotóide. Os tipos de segmentos para o alinhamento vertical previstos são linha, arco circular e parábola. A Figura 5 ilustra a conexão entre os alinhamentos horizontal e vertical na composição da posição final do espaço da infraestrutura. 


\section{$\pi$}

\section{TIC2015}

Figura 5 - Subdivisões do alinhamento para infraestruturas no IFC4.

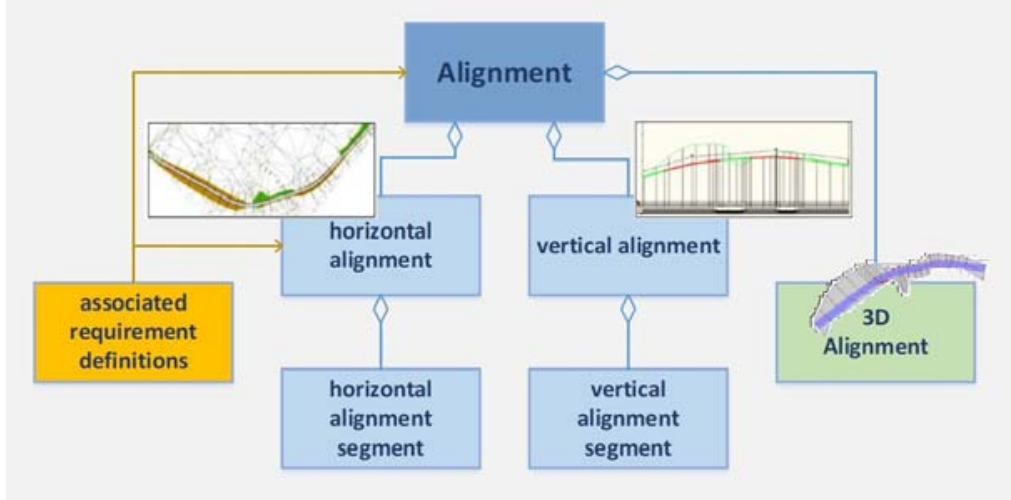

Fonte: buildingSMART, 2015c.

O desenvolvimento do IfcAlignment foi feito em conjunto com o LandInfra Group da OGC, por meio de especificação em UML (Unified Modeling Language), e depois realizado em EXPRESS e no esquema XML. Na sequência, foi definido um MVD para regulamentar as trocas de informações.

\section{A OGC E SUAS ESPECIFICAÇÕES CITYGML E INFRAGML/LANDXML}

Vale ressaltar que os esforços da buildingSMART para integrar a informação das infraestruturas no seu modelo de dados vem sendo feito consonante com os padrões já aplicados para infraestruturas no contexto dos sistemas de informação geográfica, com auxílio da OGC. A OGC é a responsável pela padronização na área de sistemas de informação geográfica.

Um de seus padrões é a Geography Markup Language (GML) (ISO, 2007). Padronizada pela ISO 19136:2007, é uma codificação em XML para o transporte e armazenamento de informações geográficas incluindo propriedades espaciais e não-espaciais de características geográficas. InfraGML e CityGML, que serão vistos mais adiante, são dois Application Schemas do GML para modelagem de infraestrutura e cidades, respectivamente.

Desenvolvido a partir do GML, e também promovido pela OGC, o CityGML define a geometria tridimensional, a topologia, a semântica e a aparência dos objetos topográficos mais relevantes do contexto urbano ou regional (GRÖGER; PLÜMER, 2012). O modelo começou a ser desenvolvido em 2002, tornou-se padrão internacional pela OGC em 2008 e, em 2012, entrou na versão 2.0. Deriva a parte de representação geométrica do GML, garantindo assim a interoperabilidade. No entanto, o CityGML tende a ser mais restrito que o GML, não possibilitando todas as suas funcionalidades no intuito de ser um modelo mais simples e funcional, do que um completo mas difícil de ser utilizado para aplicações computacionais. A geometria no CityGML é baseada na representação B-Rep.

Os objetos que fazem parte do modelo em CityGML estão agrupados em módulos: Core, Relief, Building, Tunnel, Bridge, Transportation, Water Body, Vegetation, City Furniture, Land Use, Group e Generics. 


\section{TIC2015}

Figura 6 - Os cinco níveis de detalhes existentes no CityGML.

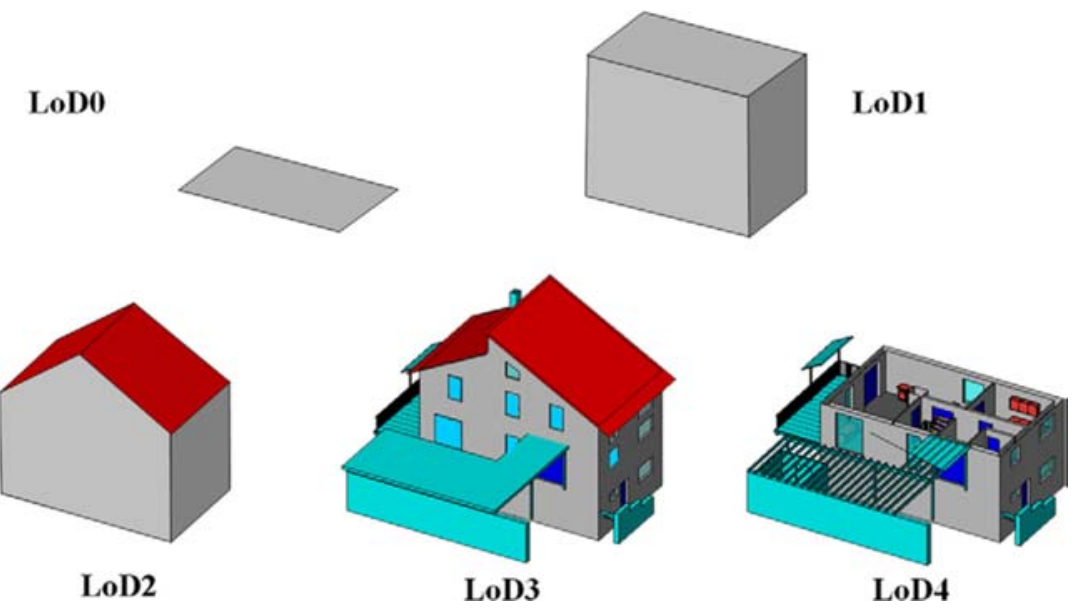

Fonte: Gröger e Plümer, 2012

O CityGML, cujo principal objeto é a edificação, possui cinco níveis de detalhe geométrico (LoD) em que cada objeto pode ser representado (ver 
Figura 6). A existência de diferentes LoD no CityGML tem como objetivo facilitar a integração de dados e a interoperabilidade num mesmo LoD, refletir requisições específicas para certas aplicações, prover uma correspondência com o método de captura de dados e facilitar tarefas de visualização e análise, permitindo que uma mesma característica possa ser representada em mais de um LoD.

O foco do modelo é na semântica dos objetos. Com a caracterização dos atributos e relações de cada tipo de objeto, diversos resultados podem ser obtidos a partir do modelo, tais como: simulação e mapeamento da propagação de ruído, aspectos energéticos de edificações, circulação no interior de edificações, gerenciamento de desastres naturais e questões de segurança nacional, dentre outros (GRÖGER; PLÜMER, 2012). Alguns trabalhos foram publicados comparando o IFC com o CityGML. A Quadro 1 apresenta as principais diferenças entre os modelos.

Quadro 1 - Comparação entre o IFC e o CityGML.

\begin{tabular}{|l|l|}
\hline \multicolumn{1}{|c|}{ IFC } & \multicolumn{1}{c|}{ CityGML } \\
\hline $\begin{array}{l}\text { Objetos semânticos focam na construção e } \\
\text { no projeto das edificações, provendo assim } \\
\text { elementos da construção tais como vigas, } \\
\begin{array}{l}\text { lajes e paredes, que tipicamente ocupam a } \\
\text { fronteira entre ambientes distintos e } \\
\text { simultaneamente são parte da fronteira } \\
\text { exterior da edificação. }\end{array}\end{array}$ & $\begin{array}{l}\text { Descreve como os edifícios são observados } \\
\text { e usados e assim os objetos parede e teto } \\
\text { são definidos para um ambiente único; }\end{array}$ \\
\hline Representação geométrica: CSG & Representação geométrica: B-Rep \\
\hline $\begin{array}{l}\text { Foco na edificação } \\
\begin{array}{l}\text { Apenas um LoD (apesar de poder conter } \\
\text { mais de uma representação geométrica) }\end{array}\end{array}$ & $\begin{array}{l}\text { Objetos relacionados à transporte, terreno, } \\
\text { ou recursos hídricos }\end{array}$ \\
\hline
\end{tabular}

Fonte: adaptado de Nagel; Stadler; Kolbe, 2009.

Uma conclusão a que se chegou é que o IFC contém toda informação necessária para construir os modelos no CityGML, independentemente do nível de detalhe demandado (ISIKDAG; ZLATANOVA, 2009).

Outro modelo que interessa no contexto em estudo é o InfraGML. Ainda não foi proposta uma versão consolidada do modelo, mas ele consistirá de um subconjunto da LandXML (CREWS et al., 2002).

O LandXML é um esquema de dados largamente utilizado pela comunidade de engenharia civil, terraplanagem e de levantamento de terrenos. Mas durante um longo período deixou de ser desenvolvido tendo voltado apenas agora em 2014, com a versão 2.0.

No entanto, sendo reconhecido que existem várias inconsistências entre o LandXML e as diretrizes da norma GML, foi criada uma comissão para, a partir da LandXML, criar um subconjunto baseado em casos de uso, que atenda aos requisitos do GML, e que irá se chamar InfraGML (ZEISS, 2013).

\section{MODELAGEM DA INFORMAÇÃO DA CIDADE OU CIM}

Por meio da modelagem da informação, promove-se um paradigma para tratar o objeto modelado em questão. Com o BIM e o IFC, tem-se uma nova maneira de projetar, construir, simular e operar uma edificação e futuramente obras de infraestrutura. Com o SIG e os CityGML e InfraGML/LandXML, tomadas de decisão e planejamento no nível de cidades vem sendo feitos com base em análises espaciais e estatísticas. A integração destes dois 
contextos, que apesar de distintos em escala, apresentam intersecções justamente nos elementos de infraestrutura, vem sendo promovida tanto por parte das universidades, quanto da indústria (KARIMI; AKINCI, 2010).

REUVERS (2014) elabora uma comparação entre o BIM e o SIG; destaca-se que o primeiro representa objetos por coordenadas espaciais (informação necessária para a geração de desenhos técnicos), enquanto o segundo, características conforme sua posição geográfica (informação necessária para a criação de mapas).

Para tratar do tema do CIM, este artigo foca no que se acredita ser seu elemento primário ou fundamental: a modelagem da informação e a possibilidade de interoperabilidade entre os sistemas que operam sobre os modelos. Para que seja desenvolvida um efetivo CIM, é necessário criar um esquema de dados, aberto, para facilitar a troca e a interoperabilidade das diversas plataformas e aplicações que irão surgir no mercado. O ecossistema computacional será mais amplo do que já existe no BIM e no GIS em separado.

Uma Modelagem da Informação da Cidade deve consistir num novo paradigma para se pensar, planejar, construir, operar e gerenciar os ativos de uma cidade. As informações referentes aos ativos devem estar devidamente representadas num modelo tridimensional e georreferenciadas, e devem servir de base para o planejamento da expansão ou recuperação dos espaços públicos, para a manutenção preventiva da infraestrutura, e como base para simular cenários com novas políticas públicas. As ferramentas computacionais associadas ao CIM constituirão uma plataforma para a transformação da cidade numa Smart City ou Cidade Inteligente.

Acredita-se que uma Modelagem da Informação da Cidade adequada permitirá a exploração da semântica envolvida para a aplicação do modelo a diversas análises e simulações. Neste contexto dois fatores são importantes:

- a aquisição de dados, principalmente relacionada à existência de uma rede de sensores que fornece informação em tempo real de aspectos chaves da cidade, como o estado do trânsito nas vias principais, a presença de vazamentos na rede de distribuição de água, sobrecarga na rede elétrica, ou mesmo o gerenciamento de uma rede elétrica do tipo smart grid, para acolher cidadãos que produzem energia maior do que o próprio consumo e o devolvem à rede;

- uma camada de processamento destes dados para transformá-los em informação e, assim, alimentar o modelo;

Com modelos que representam cidades, será fundamental que se empregue hardware e software apropriado, no que hoje é conhecido como Big Data (BERMAN, 2013).

$\mathrm{O}$ assunto $\mathrm{CIM}$, por estar diretamente relacionado às Smart Cities, é extremamente amplo e multidisciplinar. O recorte proposto neste artigo tratou apenas do modelo de dados que se acredita ser um aspecto fundamental, pois determina a semântica entre os objetos e, por conseguinte, as ações possíveis de serem executadas.

Provavelmente não será necessário criar um outro padrão para o CIM, e sim investir na integração do IFC4 (e IFC5) com o futuro modelo InfraGML e o já empregado CityGML.

\section{CONCLUSÕES}

Neste artigo foram relatados os atuais esforços da indústria e da academia para o desenvolvimento de modelos de dados para integrar as escalas micro e macro no tratamento de uma cidade. 
Discutiu-se ainda, a partir destas formas de representação, como os demais aspectos deste sistema de informações poderiam funcionar sobre os dados e assim concretizar-se como uma Modelagem da Informação da Cidade, nos moldes do paradigma BIM.

Acredita-se que o cerne de uma futura Modelagem da Informação da Cidade ou CIM deva possuir um modelo de dados padrão, consonante com o IFC ao tratar da escala da edificação e de infraestrutura, e com o InfraGML, ao tratar da escala de uma cidade. Além disso, haverá a necessidade de uma camada de processamento entre a aquisição de dados referentes ao funcionamento de uma cidade, que devem ser transformados neste modelo que o artigo discutiu. Desta forma, acredita-se que esta camada terá como base análises do tipo Big Data, incorporando técnicas das áreas de Data Analysis, Aprendizado de Máquina, e Inteligência Artificial.

Uma das conclusões deste trabalho aponta para um CIM que obtém dados externos e agrega diversos modelos BIM parciais e georreferenciados, onde o gestor da cidade aplica as ferramentas de análise espacial dos SIG sobre a informação atualizada e necessária para o planejamento urbano.

Como continuidade ao trabalho apresentado, será necessário implementar umas das muitas análises possíveis com $\circ \mathrm{CIM}$, para testar a interoperabilidade entre as informações pertinentes ao BIM e ao SIG.

\section{AGRADECIMENTOS}

Os autores agradecem ao apoio da Fundação de Amparo à Pesquisa do Estado de São Paulo (FAPESP) e o segundo autor agradece também ao CNPq - Conselho Nacional de Desenvolvimento Científico e Tecnológico.

\section{REFERÊNCIAS}

ARTHAUD, G.; LEBEGUE, E. IFC-Bridge V2 Data Model, edition R7,2002.

BERMAN, J. Principles of Big Data: preparing, sharing, and analyzing complex information. Waltham: Morgan Kaufmann, 2013. 288p.

BUILDINGSMART. IFC4. Disponível em: http://www.buildingsmart-tech.org/specifications/ifcreleases/ifc4-release/ifc4-release-summary, 2015a. Acessado em: 6 de Agosto de 2015.

BUILDINGSMART. Infrastructure. Disponível em: www.buildingsmart-tech.org/infrastructure/projects, 2015b. Acessado em: 6 de Agosto de 2015.

BUILDINGSMART. IfcAlignment. Disponível em: http://www.buildingsmarttech.org/infrastructure/projects/alignment, 2015c. Acessado em: 6 ago. 2015.

CREWS, N.; HALL, E; REBOLJ, D. LandXML Schema Version 1.0 Reference. LandXML.org.

EASTMAN, C.; et al. BIM Handbook: a guide to Building Information Modeling for owners, managers, designers, engineers, and contractors. Hoboken: John Wiley \& Sons, Inc., 2011.

FISHER, T.; MacDONALD, C. An Overview of the Canada Geographic Information System (CGIS), AutoCarto, 4, p.610-615, 1979. Disponível em:<http://mapcontext.com/autocarto/proceedings/autocarto-4-vol-1/pdf/an-overview-of-the-canada-geographic-information-system(cgis).pdf>. Acesso em: 7 ago. 2015.

GIL, J.; ALMEIDA, J.; DUARTE, J. The backbone of a City Information Model (CIM) - implementing a spatial data model for urban design. In: CONFERENCE ON EDUCATION AND RESEARCH IN COMPUTER AIDED DESIGN IN EUROPE, 2011, Proceedings ..., 2011. 
GRÖGER, G; PLÜMER, L. CityGML - Interoperable semantic 3D city models. Journal of Photogrammetry and Remote Sensing, v. 71, p. 12-33, 2012.

ISIKDAG, U.; ZLATANOVA, S. Towards defining a framework for automatic generation of buildings in CityGML using BIM. In: 3D Geo-information Sciences. Berlin: Springer pp. 79-96, 2009.

ISO - INTERNATIONAL ORGANIZATION FOR STANDARDIZATION. ISO 16739: 2013 - Industry Foundation Classes (IFC) for data sharing in the construction and facility management industries, 2013.

2007. - ISO 19136:2007 Geographic Information - Geography Markup Language (GML),

KARIMI, H.A.; AKINCI, B. CAD and GIS Integration. Boca Raton: CRC Press, 2010. 235 p.

LEE, S.-H., KIM, B.-G. IFC Extension for road structures and digital modeling. In: EAST ASIAPACIFIC CONFERENCE ON STRUCTURAL ENGINEERING AND CONSTRUCTION, 14, 2011, Proceedings..., 2011, p. 1037-1042.

LIEBICH, T. IFC4 - the new buildingSMART Standard: What's new on IFC4, 2013. Disponível em: $<$ http://www.buildingsmart-tech.org/specifications/ifc-releases/ifc4-release/buildingSMART_IFC4_ Whatisnew.pdf>. Acesso em: 14 jun 2015.

LIEBICH, T. IFC for Infrastructure - Separation between BIM and GIS requirements, 2012. Technical Report. Disponível em:

<http://www.iowadot.gov/bridge/3D/Presentations/20121017_IFC_for_Infrastructure.pdf> Acesso em: 6 de Agosto de 2015.

MOON, H. Development of IfcRoad in KICT (Korea). Disponível em: <http://iug.buildingsmart.org/ resources/international-meetings-2014-toronto/infra-room-meeting/development-ifcroad-kict>. Acesso em: 14 jun 2015.

NAGEL, C.; STADLER, A.; KOLBE, T.H. Conceptual requirements for the automatic reconstruction of Building Information Models from uninterpreted 3D models. International Archives of Photogrammetry, Remote Sensing and Spatial Information Sciences v.38, p. 46-53. 2009.

REUVERS, M. InfraGML: Linking pin BIM between Geo and BIM, 2014. Disponível em: < www.geobim.org/europe/2014/pdf/marcel_reuvers.pdf>. Acesso em 14 jun 2015.

STASCHEIT, J. et al. Process oriented numerical simulation of mechanized tunneling using an IFCbased tunnel product model. In: INTERNATIONAL CONFERENCE ON CONSTRUCTION APPLICATIONS OF VIRTUAL REALITY, 13., 2013, Londres. Proceedings..., 2013. p. 613-620.

STOJANOVSKI, T. City Information Modeling (CIM) and Urbanism: blocks, connections, territories, people and situations. In: SYMPOSIUM ON SIMULATION FOR ARCHITECTURE AND URBAN DESIGN, 4, 2013, San Diego, California, USA. Proceedings..., San Diego:ACM, 2013. .

$\mathrm{XU}, \mathrm{X}$. et al. From Building Information Modeling to City Information Modelling. Journal of Information Technology in Construction, Special Issue BIM Cloud-based Technology in the AEC Sector: Present Status and Future Trends, v.19, p. 292-307, 2014.

YABUKI, N.; et al. International Collaboration for Developing the Bridge Product Model IFC-Bridge. In: INTERNATIONAL CONFERENCE ON COMPUTING AND DECISION MAKING IN CIVIL AND BUILDING ENGINEERING, Proceedings ..., 2006.

YABUKI et al. Fundamental study on development of a shield tunnel product model. Journal of Applied Computing in Civil Engineering, v.16, p. 261-268, 2007. 
ZEISS, G. A proposal to replace LandXML with a new standard InfraGML. 10 Dez. 2013. Disponível em:<http://geospatial.blogs.com/geospatial/2013/12/a-proposal-to-replace-landxml-withinfragml.html>. Acesso em 14 jun. 2015. 